\title{
Nonconventional and new methods in the diagnosis of tuberculosis: feasibility and applicability in the field
}

\author{
J.C. Palomino
}

ABSTRACT: Tuberculosis (TB) remains one of the major causes of death from a single infectious agent worldwide. Of great concern for TB control is the emergence of drug resistance. Since there is no cure for some multidrug-resistant strains of Mycobacterium tuberculosis, there is concern that they may spread around the world, stressing the need for additional control measures, such as new diagnostics, better drugs for treatment, and a more effective vaccine.

Pulmonary TB can be diagnosed by its symptoms, chest radiography, sputum smear microscopy and by cultivation of $M$. tuberculosis, which is considered as the gold standard. Recent advances in molecular biology and molecular epidemiology, and a better understanding of the molecular basis of drug resistance in TB, have provided new tools for rapid diagnosis; however, the high cost of most of these techniques, and their requirement for sophisticated equipment and skilled personnel have precluded their implementation on a routine basis, especially in low-income countries.

Other nonconventional diagnostic approaches proposed include the search for biochemical markers, detection of immunological response and early detection of $\boldsymbol{M}$. tuberculosis by methods other than colony counting.

In the present article, some of these approaches will be reviewed and the feasibility for their implementation in diagnostic laboratories will be discussed.

KEYWORDS: Diagnosis, drug resistance, tuberculosis

$\mathbf{T}$ uberculosis (TB) remains one of the major causes of death from a single infectious agent worldwide. A total of $99 \%$ of the estimated two million deaths and $95 \%$ of the more than eight million new cases each year occur in middle- and low-income countries. The World Health Organization (WHO) report [1] estimated, for the year 2002, a global incidence of 8.8 million new cases, including 3.9 million smear-positive subjects. The 22 high-burden countries concentrated $80 \%$ of these cases. This situation is worsened by the HIV pandemic, since the risk of death in HIV-infected patients with TB is twice that of HIV-infected patients without TB [2]. It is estimated that 12 million patients are coinfected with HIV and Mycobacterium tuberculosis as of 2000, with the majority living in subSaharan Africa and Southeast Asia.

Of great concern for the control of the disease is the emergence of drug resistance (DR) since there is no cure for some multidrug-resistant (MDR) strains of $M$. tuberculosis, and there is concern that they may spread rapidly around the world. DR

Previous articles in this series: No. 1: Cardona P-J, Ruiz-Manzano J. On the nature of Mycobacterium tuberculosis-latent bacilli. Eur Respir J 2004; 24 1044-1051. No. 2: Rieder H. Annual risk of infection with Mycobacterium tuberculosis. Eur Respir J 2005; 25: 181-185. No. 3: Mitchison DA. Drug resistance in tuberculosis. Eur Respir J 2005; 25: 376-379. No. 4: Kim SJ. Drug-susceptibility testing in tuberculosis: methods and reliability of results. Eur Respir J 2005; 25 : 564-569. No. 5: Dlodlo RA, Fujiwara PI, Enarson DA. Should tuberculosis treatment and control be addressed differently in HIV-infected and -uninfected individuals? Eur Respir J 2005; 25: 751-757. No. 6: Caminero JA. Management of multidrug-resistant tuberculosis and patients in retreatment. Eur Respir J 2005; 25: 928-936. No. 7: Dasgupta K, Menzies D. Cost-effectiveness of tuberculosis control strategies among immigrants and refugees. Eur Respir J 2005; 25: 11071116. No. 8: Martín C. The dream of a vaccine against tuberculosis; new vaccines improving or replacing BCG? Eur Respir J 2005; 26: 162-167.

CORRESPONDENCE

J.C. Palomino

Mycobacteriology Unit Institute of Tropical Medicine Nationalestraat 155 2000 Antwerp Belgium Fax: 3232476333 E-mail: palomino@itg.be

Received:

April 262005 Accepted: April 282005

\section{SUPPORT STATEMENT}

Financial support was provided by the EC INCO Programme (Contract No. ICA4-CT-2001-10087), EC LIFESCIHEALTH-3 (Contract No. 516028), Damien Foundation (Brussels, Belgium) and Fund for Scientific Research (Flanders, Belgium) 
has been found in all countries and regions surveyed, particularly in Eastern Europe, and new areas with high prevalence of MDR-TB include China and Iran [3]. Directlyobserved treatment short course (DOTS), the treatment strategy endorsed by the $\mathrm{WHO}$, is effective in preventing the emergence of DR; however, in practice, only $27 \%$ of TB patients actually receive DOTS [4], stressing the need for additional control measures, such as new diagnostic tools, more effective drugs, or even a more effective vaccine.

Pulmonary TB, the most important type of TB from the public health point of view, can be diagnosed by its symptoms, chest radiography, sputum smear microscopy, and by cultivation of M. tuberculosis. A percentage of patients, however, are not confirmed bacteriologically and are only diagnosed on the basis of high clinical suspicion and response to anti-TB drugs [5].

The gold standard for TB diagnosis is the cultivation of M. tuberculosis. It can be performed on a variety of specimens, such as sputum and bronchial washings, and also other nonpulmonary samples. It is much more sensitive than microscopy and it allows the recovery of the bacteria for other studies, such as drug susceptibility testing and genotyping. In some cases, the diagnosis of TB becomes even more problematic due to several factors associated with immunosuppresion in patients as it occurs in HIV infected persons or in the case of latent infection or extrapulmonary TB. Due to its nonspecific clinical presentation, diagnosis of $\mathrm{TB}$ is also problematic in children.

Recent advances in the field of molecular biology and progress in the understanding of the molecular basis of DR in M. tuberculosis have provided new tools for its rapid diagnosis by molecular methods [6]. However, the high cost of most of these techniques, and their requirement for sophisticated equipment or highly skilled personnel, have precluded their implementation on a routine basis, especially in low-income countries [7]. Other nonconventional approaches recently proposed include the search for biochemical markers, detection of immunological response and early detection of M. tuberculosis by methods other than colony counting. In the present article, some of these approaches will be reviewed and the feasibility for their implementation in diagnostic laboratories will be discussed.

\section{NEW METHODS FOR THE DETECTION OF TB INFECTION}

Since one-third of the worlds population is infected with M. tuberculosis, it would be important to be able to predict who among the latently infected will develop the disease, so as to treat them before active TB is developed. The only available test to detect infection was, until recently, the tuberculin skin test (TST). However, alternative in vitro T-cell-based methods have been proposed recently [8-10]. The interferon (IFN) $-\gamma$ assays are based on the fact that T-cells sensitised with tuberculous antigens will produce IFN- $\gamma$ when they are re-exposed to mycobacterial antigens. A high amount of IFN- $\gamma$ production is then presumed to correlate with TB infection [11]. The first IFN- $\gamma$ assays made use of purified protein derivative (PPD) as the stimulating antigen; more recent assays, use antigens that are specific to $M$. tuberculosis, such as the early secretory antigen target 6 (ESAT6), and the culture filtrate protein 10 (CFP10) [8]. These protein antigens are coded by genes located in the region of difference 1 (RD1) of the $M$. tuberculosis genome and are much more specific than PPD, since they are not shared with $M$. bovis bacillus calmette-guerin (BCG) or most nontuberculous mycobacteria (NTM) with the exception of M. marinum, M. sulzgai and M. kansassi.

There are currently two commercial IFN- $\gamma$ assays, the QuantiFERON-TB assay (Cellestis Ltd., Carnegie, Australia) and the T SPOT-TB test (Oxford Immunotec, Oxford, UK). These tests measure the production of IFN- $\gamma$ by T-cells in response to $\mathrm{TB}$ antigens by ELISA and enzyme-linked immunospot, respectively. The QuantiFERON-TB, a wholeblood assay, initially used PPD as antigen, and the enhanced version, the QuantiFERON-TB Gold, uses ESAT6 and CFP10. In contrast, the T SPOT-TB assay uses peripheral blood mononuclear cells and ESAT6 and CFP10 as antigens to measure the number of T-cells producing IFN- $\gamma$. Also, some noncommercial in-house methods have been proposed [12].

In general, the studies performed have shown that IFN- $\gamma$ assays using RD1 antigens have some advantages over TST, such as a higher specificity, a better correlation with previous exposure to $M$. tuberculosis, and low cross-reaction due to BCG vaccination or previous exposure to NTM. Also, it has been found that IFN- $\gamma$ assays that use cocktails of antigens rather than individual antigens have a better accuracy [13]. More studies are needed, however, to assess the usefulness of these tests in the management of immunocompromised individuals, in children and in those with extrapulmonary TB.

\section{NONCONVENTIONAL AND NEW METHODS OF BACILLOSCOPY}

In many countries diagnosis of TB is performed by microscopic examination of a stained sputum smear by the Ziehl-Neelsen (ZN) method. Although easy to perform and specific, it lacks sensitivity, requiring $\geqslant 10,000$ bacilli $\cdot \mathrm{mL}^{-1}$ of sputum to become positive. Several studies have been performed to assess the usefulness of adding a chemical reagent, such as sodium hypochlorite, to liquefy and then concentrate the sputum by further centrifugation to increase sensitivity. In most of these studies, a statistically significant improvement in the proportion of positive smears or sensitivity was obtained; however, for several reasons, sodium hypochlorite, also known as the "bleach" method is not used routinely in many settings [14]. The use of auramine as a fluorescent method to detect mycobacteria in sputum was proposed many years ago and re-evaluated later using a combination of auramine-O and rhodamine [15]. This fluorescent method is associated with a higher rate of detection, since slides can be examined at lower magnifications. In a recent proficiency testing study performed in 167 laboratories, the auramine and/ or the auramine/rhodamine method performed better than the $\mathrm{ZN}$ staining or its Kinyoun modification [16]. It is, therefore, usually accepted that the fluorescent method should be given preference over the $\mathrm{ZN}$ and Kinyoun methods. In general, the fluorescent method should be used by laboratories with large specimen numbers. It is, however, more expensive than the conventional $\mathrm{ZN}$ staining requiring a fluorescent microscope [17]. 


\section{NONCONVENTIONAL AND NEW METHODS OF CULTURE}

Cultivation of $M$. tuberculosis from clinical samples is the gold standard for the diagnosis of active TB. It can detect 100 bacilli $\cdot \mathrm{mL}^{-1}$ of sputum in comparison with 5,000 10,000 bacilli $\cdot \mathrm{mL}^{-1}$ needed for microscopy [18]. It also provides material for further identification and drug susceptibility testing. Conventional methods of culture have relied on eggbased and agar-based media, such as the Löwenstein-Jensen (LJ) medium and Middlebrook agar [19, 20]. Following decontamination and liquefaction procedures, sputum samples are inoculated and incubated for morphological growth, which usually occurs after several weeks of incubation. Identification of $M$. tuberculosis is done by performing several further biochemical tests $[19,21]$. However, it is laborious and time consuming requiring from 3-8 weeks to obtain the results.

The introduction of the BACTEC radiometric system (BACTEC TB-460; Becton Dickinson, Sparks, MD, USA) in the 1980s was a breakthrough since it allowed the detection of $M$. tuberculosis in a few days compared with weeks in the conventional culture media [22]. However, the use of radioisotopes and the cost of the equipment precluded its use on a routine basis, except in reference laboratories predominantly in developed countries. A few years ago Becton Dickinson proposed another system based on fluorescence detection of mycobacterial growth [23]. The Mycobacteria growth indicator tube (MGIT) system is based on a glass tube containing a modified Middlebrook 7H9 broth together with a fluorescence quenching-based oxygen sensor embedded at the bottom of the tube. When inoculated with $M$. tuberculosis, consumption of the dissolved oxygen produces fluorescence when illuminated by a UV lamp. The MGIT system has been thoroughly evaluated in clinical settings for the detection and recovery of mycobacteria. BADAK et al. [24] compared the MGIT system with the BACTEC TB-460 and LJ culture medium in 1,441 clinical specimens. Out of 178 isolates recovered, $30(17 \%)$ were M. tuberculosis with the MGIT system recovering $28(93 \%)$ compared with $25(83 \%)$ recovered with the LJ medium. In another multicentre study, PFYFFER et al. [25] analysed 1,500 clinical specimens detecting a total of 180 mycobacterial species comprising $113 \mathrm{M}$. tuberculosis complex isolates. The combination of MGIT and BACTEC detected 171 (95\%) of all isolates with a time to detection of $M$. tuberculosis of 9.9 days compared with 9.7 days with BACTEC and 20.2 days with solid medium proving that MGIT was a valuable alternative to the radiometric system [25].

More recently the MGIT system has been fully automated and turned into the BACTEC MGIT 960 system, which is a nonradiometric, noninvasive system with the tubes incubated in a compact system that reads them automatically. In a multicentre study the BACTEC MGIT 960 system was compared with the radiometric BACTEC TB-460 system and LJ medium. Analysing 2,576 specimens, the best yield was obtained with BACTEC TB-460 (201 isolates), compared with 190 isolates with BACTEC MGIT 960 and 168 isolates with LJ medium [26]. In another study IDIGORAS et al. [27] compared the BACTEC MGIT 960 system for sensitivity and time to detection of mycobacteria with solid medium, and microscopy on solid media. Sensitivity of each media compared with all media combined for growth of $M$. tuberculosis was $93 \%, 76 \%, 79 \%$ and $75 \%$ for MGIT 960, Middlebrook 7H11, LJ and Coletsos, respectively. The time to detection with the MGIT 960 system was 12.7 days compared with $>20$ days with the solid media. In general, both the automated and manual MGIT systems have shown similar results that are comparable to those obtained previously with the BACTEC radiometric method. Operational and cost-effectiveness studies that assess the real impact of these systems in low- and middle-income countries are still lacking.

Other recent developments for the rapid detection of mycobacteria include manual methods like the MB-Redox (Heipha Diagnostika Biotest, Heidelberg, Germany) based on the reduction of a tetrazolium salt indicator in liquid medium [28], and automated equipment-based methods like the MB/ BacT system (Organon Teknika, Boxtel, Holland) based on the colorimetric detection of carbon dioxide produced by mycobacterial growth in a closed system [29], and the ESP culture system II (Trek Diagnostics, Inc., Cleveland, OH, USA) based on the detection of pressure changes in the culture medium of a sealed vial during mycobacterial growth [30]. These systems have not gained widespread use outside laboratories in industrialised countries.

Another recent and interesting development is the phagebased assay that relies on the ability of $M$. tuberculosis to support the growth of an infecting mycobacteriophage. The number of endogenous phages, representing the original number of viable $M$. tuberculosis, is then determined in a rapidly-growing mycobacteria, such as $M$. smegmatis [31]. The FASTPlaque TB assay (BIOTEC, Ipswich, Suffolk, UK), a commercial test based on this technology, has been proposed as a test that if used in conjunction with smear microscopy could increase the diagnosis of TB [32]. Several studies have been made to assess this technology. Albert et al. [32] in a comparative study with auramine smear microscopy and LJ medium in 1,692 sputum specimens found that the FASTPlaque TB test detected TB in $75 \%$ of cultureconfirmed cases and $70 \%$ of cases with a clinical diagnosis of TB, with a specificity of $98.7 \%$ and $99.0 \%$, respectively. In contrast, the concentrated auramine smear microscopy had a sensitivity of $63.4 \%$ and $61.3 \%$ and a specificity of $97.4 \%$ and $97.3 \%$ in culture- confirmed and all cases, respectively [32].

Another study performed in Pakistan compared the FASTPlaque TB with acid-fast smear microscopy and culture in LJ medium [33]. For the FASTPlaque TB, they found a sensitivity and specificity of 87.4 and $88.2 \%$, respectively, in smear-positive specimens and a sensitivity and specificity of 67.1 and $98.4 \%$, respectively, in smear-negative samples. As discussed by TAKIFF and HEIFETS [34], the most important finding of these studies was that the FASTPlaque TB was able to detect mycobacteria in $50-65 \%$ of smear-negative specimens with a specificity of $98 \%$ and that a combination of the test with smear microscopy confirmed the presence of $M$. tuberculosis in $80-90 \%$ of culture-positive specimens. Still, there were about $13 \%$ of smear-positive specimens that were not detected by the FASTPlaque TB test and in smear-negative samples $8-19 \%$ of specimens gave a false-positive result.

More recently, in a comparative study of the FASTPlaque TB test and the original in-house method performed in Zambia, 
MBULO et al. [35] found that neither method was able to outperform direct microscopy in sputum samples. Furthermore, contamination rates of $40 \%$ were obtained with the FASTPlaque TB test, concluding that the phage-based assays offered no advantage for TB diagnosis in that setting. Some other phage-based technologies have been proposed for the rapid detection of $M$. tuberculosis [36, 37]; however, they have not been thoroughly evaluated in a clinical setting in highendemic countries.

\section{NONCONVENTIONAL METHODS AND NEW METHODS FOR IDENTIFICATION}

Mycobacteria identification has been based traditionally on several biochemical tests and phenotypic characteristics, such as growth rate and pigmentation, which allow the classification of one particular strain to a group of well-defined mycobacteria. Although simple to perform and without the need for sophisticated equipment, they are, nonetheless, laborious and cumbersome to perform, delaying in many cases prompt and correct mycobacterial identification. Nevertheless, they remain and constitute the main procedure for identification in clinical laboratories, especially in lowresource settings. Mycolic acid profiles of mycobacteria were also proposed as a rapid alternative for identification. This can be performed by thin-layer chromatography and by highperformance liquid chromatography, which has the advantage that it can be completed in a few hours, it is relatively inexpensive, and can identify a wide range of mycobacterial species. The initial investment in the cost of the equipment constitutes its major disadvantage as an identification method [38].

Molecular methods have been more recently introduced for identification of mycobacteria. The first of such available methods was the AccuProbe (Gen-Probe, Inc., San Diego, CA, USA), a commercial method based on species-specific DNA probes that hybridise to rRNA for the identification of several important mycobacteria, including the M. tuberculosis complex, M. avium, M. intracellulare, M. avium complex, M. kansasii, and M. gordonae. The probes have been extensively evaluated in a clinical setting, and have shown very good sensitivity and specificity giving results in $\sim 2 \mathrm{~h}$ from culture-positive specimens [39].

Other commercially available methods include the INNO-Lipa Mycobacteria (LiPA; Innogenetics, Ghent, Belgium) for the simultaneous detection and identification of mycobacteria, including the M. tuberculosis complex and based on amplification of the 16S-23S spacer region combined with a reverse hybridisation line-probe assay. It has been evaluated against DNA probes, conventional biochemical tests, and PCRrestriction fragment length polymorphism analysis showing clear-cut results $[40,41]$

More recently the GenoType Mycobacterium assay (Hain Diagnostika, Nehren, Germany) for identification from clinical samples and liquid cultures has been introduced. It is based on amplification of the 16S-23S ribosomal DNA spacer region followed by hybridisation with 16 specific oligonucleotide probes; it has the advantage of detecting mixed mycobacterial infections [42].

A commercial system designed primarily for culture confirmation, TB peptide nucleic acid fluorescence in situ hybridisation
(Dako, A/S Glostrup, Denmark) has also been proposed. It is based on peptide nucleic acid probes that bind to selected regions of mycobacterial 16S rRNA sequences allowing distinction between tuberculous and NTM; detection is done by fluorescence in situ hybridisation followed by microscopic observation [43]. It has also been tested for the direct detection of $M$. tuberculosis on smear-positive sputum samples and on formalin-fixed, paraffin-embedded biopsies [44].

PCR-based sequencing techniques are now common in many laboratories for the identification of mycobacteria. They consist of amplification of mycobacterial DNA with genus-specific primers followed by sequencing of the amplified products. Identification is done by comparison of the sequences with those of reference strains $[45,46]$. The most commonly used target has been the gene coding for the 16S rRNA, although other target genes have also been characterised [47, 48].

PCR-restriction fragment length polymorphism analysis (PRA) of the gene coding for the heat shock protein hsp65 has also been used for the rapid identification of mycobacteria, including $M$. tuberculosis [49]. In several recent studies PRA has performed well compared with conventional methods [50, 51].

More recently, DNA microarrays or high-density oligonucleotide arrays have been applied for species identification of mycobacteria. The method is based on hybridisation of fluorescently-labelled PCR amplified products obtained from mycobacterial colonies to DNA arrays containing nucleotide probes $[52,53]$.

In general, molecular methods offer several advantages over conventional techniques for the rapid detection and identification of M. tuberculosis, such as the turnaround time for results, reliability, reproducibility and the possibility to improve patient management. However, due to the requirement of additional equipment and trained personnel, most of these methods have not yet gained easy access to the routine procedures performed in the clinical mycobacteriology laboratory especially in low-income countries where TB is a more important health problem.

\section{NONCONVENTIONAL AND NEW METHODS FOR DRUG SUSCEPTIBILITY TESTING}

Drug susceptibility testing (DST) methods include the proportion method, the absolute concentration method, and the resistance ratio method $[19,54]$. With the introduction of the BACTEC radiometric system, and its adaptation to perform DST of $M$. tuberculosis [22, 55], these four methods were considered as the gold standard. [56]. The long turnaround time (TAT) and laboriousness of these methods stimulated the search for alternative and faster techniques. These new methods can be differentiated into genotypic and phenotypic methods.

\section{Genotypic methods}

These methods search for the genetic determinants of resistance rather than the resistance phenotype. In general, they have two basic steps: a molecular nucleic acid amplification step, such as PCR to amplify sections of the M. tuberculosis genome, known to be altered in resistant strains and a second step of assessing the amplified products for specific mutations 
correlating with resistance. These methods have several advantages: a faster TAT (days instead of weeks), no need for growth of the organism, the possibility for direct application to clinical specimens, reduction of biohazard risks, and feasibility for automation. Unfortunately, they also have disadvantages, including problems with inhibitors when applying these methods directly to clinical samples.

DNA sequencing of PCR-amplified products has been the most widely used method, becoming the gold standard. It has been performed by both manual and automated procedures although the latter has been the most commonly used [5759]. It has been thoroughly used for characterising mutations in the rpoB gene in rifampicin-resistant strains and to detect mutations responsible for resistance to other anti-TB drugs [57, $60,61]$. Several other genotypic methods have been proposed to detect resistance to antibiotics, such as PCR-single strand conformation polymorphism [57], PCR-heteroduplex formation [62], and solid-phase hybridisation assays. Moreover, the Line Probe Assay (LiPA-Rif) based on the hybridisation of amplified DNA from cultured strains or clinical samples to ten probes encompassing the core region of the rpoB gene of M. tuberculosis, immobilised on a nitrocellulose strip [63], and the GenoType mycobacterium tuberculosis test (MTB) DR (Hain, Germany), a commercial system for the detection of the $M$. tuberculosis complex and its resistance to rifampicin and isoniazid from culture samples based on the detection of the most common mutations in the rpoB and katG genes, respectively. The LiPA-Rif assay has been evaluated in different settings giving encouraging results [64].

DNA microarrays and real-time PCR techniques have also been proposed as alternative methods for drug resistance detection; the former still beyond the reach of clinical diagnostic laboratories, and the latter being increasingly evaluated with promising results $[65,66]$.

\section{Phenotypic methods}

New phenotypic methods assess inhibition of M. tuberculosis in the presence of antibiotics by detecting earlier signs of growth using various technologies, for example, the measurement of metabolism with the aid of colour indicators, or oxygen consumption, by early visualisation of micro-colonies, and by the use of phages.

The MGIT system, in its manual or automated version and based on the measurement of oxygen consumption, has been thoroughly evaluated for DST of $M$. tuberculosis to first- and second-line drugs showing a good concordance with the gold standard proportion method [67-69].

The E-test, another commercial system (AB BIODISK, Solna, Sweden), based on strips with impregnated gradients of antibiotics for the determination of drug susceptibility allows the reading of minimal inhibitory concentrations directly on agar plates. Several studies have evaluated this test in comparison with the proportion method finding an agreement of $>90 \%$ [70]. Two other commercial and automated methods for DST are the MB/BacT system (Organon Technika) and the ESP culture system II (Accumed International, Chicago, IL, USA). Both systems rely on heavy equipment and have also been evaluated in several studies [71, 72].
Among the in-house methods of recent application for DST of M. tuberculosis, three types of methods deserve a mention: 1) phage-based assays; 2) colorimetric methods; and 3) the nitrate reduction assay.

Application of phage-based assays for DST, either as the original in-house method [73] or as a commercial assay [74], is being applied for detecting rifampicin resistance of M. tuberculosis both in culture samples and directly from sputum. Results are available, on average, in 2 days with a concordance of $>95 \%$ when compared with the gold standard proportion method.

Colorimetric methods are based on the reduction of a coloured indicator added to the culture medium after $M$. tuberculosis has been exposed in vitro to different antibiotics. Resistance is detected by a change in the colour of the indicator, which is directly proportional to the number of viable mycobacteria in the medium [75]. Different indicators have been evaluated for DST of first- and second-line drugs giving comparable results and are in agreement with the gold standard proportion method [76-78].

The nitrate reduction assay is a very simple technique based on the capacity of $M$. tuberculosis to reduce nitrate to nitrite, which is detected by adding a chemical reagent to the culture medium. M. tuberculosis is cultivated in the presence of the antibiotic and its ability to reduce nitrate is measured after 10 days of incubation. Resistant strains will reduce the nitrate revealed by a pink-red colour in the medium, while susceptible strains will loose this capacity as they are inhibited by the antibiotic, leaving the medium colourless [79]. The test has been recently evaluated for DST to first- and second-line drugs with good results $[80,81]$. It has the added advantage of using the same format and culture medium as used in the standard proportion method.

In their current format, colorimetric methods seem more appropriate for reference laboratories with the facilities and biosafety conditions to manipulate small volumes of liquid cultures in a microplate format. The phage-based assay can be implemented in mycobacteriology diagnostic laboratories with the appropriate trained personnel, while the nitrate reduction assay is ideal for implementation in the TB diagnostic laboratories routinely performing DST.

Table 1 shows the major DST methods in terms of accuracy, cost, ease of use and drugs evaluated.

\section{NUCLEIC ACID AMPLIFICATION METHODS}

A significant improvement in the diagnosis of TB was the development of several nucleic acid amplification (NAA) techniques, such as PCR, that has been extensively evaluated for the rapid diagnosis of TB. Several in-house PCR methods have been developed and tested in the last years and multiple studies have been published on the application of PCR for the diagnosis of TB $[82,83]$. Those studies found that lack of specificity was more of a problem than lack of sensitivity, and it was not associated with the use of any particular method. It was also found that many laboratories did not use adequate quality controls [84, 85].

There are currently two approved commercial NAA methods: the amplified M. tuberculosis direct test (AMTD) (Gen-Probe) 


\begin{tabular}{|c|c|c|c|c|}
\hline Method & Accuracy \% & Cost & Skills/equipment & Coverage \\
\hline BACTEC radiometric & $>95$ & High & Yes & Most drugs \\
\hline Manual MGIT & $>90$ & Medium & No & Several drugs \\
\hline Automated MGIT 960 & $>90$ & High & Yes & $\mathrm{R}, \mathrm{H}, \mathrm{E}, \mathrm{S}$ \\
\hline DNA sequencing & $>90$ (for $\mathrm{R}$ ) & High & Yes & Several drugs \\
\hline Solid-phase hybridization test & $>95$ & High & Yes & R \\
\hline FastPlaque TB & $>90$ & Low/medium & Some skill & $\mathrm{R}, \mathrm{H}$ \\
\hline MB/BacT & $>95$ & High & Yes & $R, H, E, S$ \\
\hline ESP System II & $>93$ & High & Yes & $R, H, E, S$ \\
\hline Colorimetric methods & $>95$ & Low & No & Most drugs \\
\hline
\end{tabular}

MGIT: mycobacteria growth indicator tube; TB: tuberculosis; R: rifampicin; H: isoniazid; E: ethambutol; S: streptomycin.

and the Amplicor M. tuberculosis test (Amplicor; Roche Diagnostic Sytems, Inc., NJ, USA). The MTD is based on a method described by $\mathrm{KwOH}$ et al. [86] using amplification of $16 \mathrm{~S}$ ribosomal transcripts, which are detected with a DNA probe [87]. The Amplicor is a DNA-based test which amplifies the 16S rRNA gene using genus-specific primers and detected in a colorimetric reaction [88]. Both methods have been approved for the direct detection of $M$. tuberculosis in smearpositive respiratory specimens. More recently, an enhanced MTD has also been approved for smear-negative respiratory specimens of suspect patients [89]. Several studies have evaluated both tests for the detection of M. tuberculosis in clinical samples [90]. Compared with culture and the clinical status, these methods have a high sensitivity and specificity in smear-positive specimens, but lower values are obtained in smear-negative specimens precluding their use as a screen to rule out the disease. For this reason, it has been recommended that molecular methods should always be interpreted in conjunction with the patient's clinical data [91].

Two other methodologies recently introduced for TB diagnosis involve real-time PCR and strand-displacement amplification methods. The real-time PCR technology is based on hybridisation of amplified nucleic acids with fluorescent-labelled probes spanning DNA regions of interest and monitored inside thermal cyclers [92]. The fluorescent signal increases in direct proportion to the amount of amplified product in the reaction tube.

Real-time PCR has been evaluated in several studies in culture material and more recently in clinical samples. The sensitivity in these studies has ranged from 71-98\%, with specificity close to $100 \%$ [93]. The main advantage of real-time PCR is its speed in giving results, $1.5-2 \mathrm{~h}$ after DNA extraction, and the decrease in the risk of contamination since both reaction and detection occurs in a single tube. Further studies are necessary to confirm the real value of this new methodology in the clinical setting.

The strand-displacement amplification technique is used by the BDProbe Tec MTB test, a semi-automated system developed by Becton Dickinson for the rapid detection of $M$. tuberculosis in respiratory samples. It is based on the enzymatic replication of target sequences of the insertion sequence IS6110 and the 16S rRNA gene. The amplified products are detected with a luminometer [94]. The BDProbe Tec MTB test has been evaluated in several studies; PFYFFER et al. [95], in a study with 799 respiratory specimens, obtained an overall sensitivity of $97.6 \%$ and a specificity of $95 \%$. The major drawbacks, however, have been the presence of false-positive results, that were reduced after the personnel gained experience with the technique, and the time required for sample preparation being $\geqslant 2 \mathrm{~h}$. An improved version of this system, the BDProbe Tec ET (Becton Dickinson), has been recently evaluated in respiratory specimens in a clinical setting [96]. Studies including more positive TB patients are needed to confirm these preliminary results. Table 2 shows sensitivity and specificity values of the main NAA tests.

\section{MOLECULAR EPIDEMIOLOGY TECHNIQUES}

DNA fingerprinting techniques include the restriction fragment length polymorphism (RFLP) typing as the most commonly used method in the study of the epidemiology and pathogenesis of TB. It is based on the insertion sequence IS6110 present in M. tuberculosis and is accepted as the standard typing method [97]. RFLP has been used to differentiate strains of $M$. tuberculosis, for monitoring transmission, to define strain clusters within populations, to differentiate between exogenous re-infection and relapse, to identify laboratory cross-contaminations, to study molecular evolution at the species level and for better understanding the pathogenesis of the disease.

Another molecular typing technique widely used is the spacer oligotyping or spoligotyping, which is a simple method allowing simultaneous detection and typing of $M$. tuberculosis in clinical samples. It is based on polymorphism of the chromosomal DR locus that contains a variable number of short direct repeats interspersed with nonrepetitive spacers. Most clinical isolates show unique patterns of hybridisation, while strains from a cluster share the same pattern [98]. 
TABLE 2 Sensitivity and specificity of nucleic acid amplification methods in different samples\#

\begin{tabular}{|c|c|c|c|c|c|c|c|c|}
\hline & \multicolumn{2}{|c|}{ Amplicor } & \multicolumn{2}{|c|}{ AMTD } & \multicolumn{2}{|c|}{ Real-time PCR } & \multicolumn{2}{|c|}{ BDProbeTec $^{+}$} \\
\hline & Sensitivity & Specificity & Sensitivity & Specificity & Sensitivity & Specificity & Sensitivity & Specificity \\
\hline Smear positive pulmonary & 97 & $>95$ & $92-100$ & $>95$ & 78 & 100 & $90-100$ & 92 \\
\hline Smear negative pulmonary & $40-73$ & $>95$ & $40-93$ & $>95$ & 78 & 100 & $33-100$ & $83-97$ \\
\hline Extrapulmonary & $27-98$ & $>95$ & 93 & $>95$ & 80 & 100 & 76 & $>90$ \\
\hline
\end{tabular}

Data are presented as \%. AMTD: amplified Mycobacterium tuberculosis direct test. \#: adapted from references [22] and [91]; ": Amplicor M. tuberculosis test; ${ }^{+}:$amplified M. tuberculosis direct test.

Polymorphic GC-rich repetitive sequence (PGRS) typing has also been used for this purpose [99]. PGRS is the most abundant repetitive element in the $M$. tuberculosis complex that is present in numerous copies and consists of tandem repeats of a 96 base pair (bp) GC-rich sequence. PGRS typing is mostly used as a secondary typing method of strains with a low copy number of IS6110.

More recently, two other PCR-based techniques have been developed for molecular typing: genomic deletion analysis and mycobacterial interspersed repetitive units (MIRU) typing [100, 101]. Genomic deletion analysis uses DNA microarrays to detect genomic deletions relative to a reference strain of M. tuberculosis. It can be used to provide insights into the epidemiology, genomic evolution, and population structure of M. tuberculosis.

The MIRU typing is based on the variability in the numbers of tandem repeats that are $40-100 \mathrm{bp}$ elements dispersed in intergenic regions of the $M$. tuberculosis genome; a total of 41 loci have been identified in M. tuberculosis with 12 of them showing polymorphism. This typing technique has been compared with RFLP typing and spoligotyping producing more distinct patterns [102]. It is currently accepted that MIRUbased techniques will eventually replace classical IS6110 RFLP typing once a standardised protocol has been developed [103].

\section{SEROLOGICAL DIAGNOSIS OF TB}

An important issue for the control of TB is the ability for diagnosis and treatment of latent infection. The traditional method to assess this has been the TST. The PPD utilised in the TST has been used for 50 yrs to diagnose TB in the clinic and for screening in TB control programmes for epidemiological purposes [8]. PPD is a crude mixture of antigens with the limitation that some of them are shared among $M$. tuberculosis, M. bovis BCG and some NTM. Due to this, TST has a low specificity in BCG-vaccinated populations and those previously exposed to NTM [11]. Sensitivity can also be low in immunosuppresed individuals, such as those infected with HIV.

Different types of blood tests have been proposed for serologic diagnosis of TB [5, 104, 105]. The first studies using partially purified antigens allowed the detection of anti-mycobacterial antibodies in TB patients, but the tests showed poor specificity [106]. The use of highly purified native or recombinant antigens increased specificity, but decreased sensitivity. It has also been found that the degree of humoral response to TB is heterogeneous [107]; for this reason, the use of serodiagnostic tests based on mixtures of multiple $M$. tuberculosis antigens has been proposed [108]. However, until now, none of these tests have shown to be predictive enough to warrant their routine use as diagnostic tests for TB.

\section{OTHER NON-MICROBIOLOGICAL DIAGNOSTIC TECHNIQUES}

Among the non-microbiological diagnostic techniques applied for tuberculosis, the detection of adenosine deaminase (ADA) has received most attention as an indirect method of diagnosis. ADA is an enzyme present in most cells, but has been found to be increased in tuberculous pleural effusions (TPE). Diagnosis of TPE is difficult due to the low sensitivity of direct microscopy and culture. Furthermore, lymphocytic exudates can occur in other diseases, such as malignancy and systemic lupus erythematosus [109]. Determination of ADA levels has been praised as a promising marker since it can be performed easily, rapidly and at a low cost by a colorimetric method. LEE et al. [110] have shown that ADA levels found in other groups of patients did not exceed the diagnostic cut-off level for TPE. Other studies have assessed the usefulness of measuring ADA levels in cerebrospinal fluid for diagnosis of tuberculous meningitis, and in serum for diagnosis of pulmonary TB. These studies showed that measuring ADA levels was not a good marker for diagnosis [111, 112].

More recently, the combined use of PCR and ADA for the diagnosis of TPE in a region of high prevalence of TB allowed confirmation of the disease in 14 out of 16 positive patients. As recommended, ADA should not be used to replace current diagnostic methods, but as an extra tool in the diagnosis [113].

\section{IMPLEMENTATION AND PERSPECTIVES FOR NEW DIAGNOSTIC METHODS}

Several new diagnostic approaches have been proposed for TB and several others will surely appear in the near future. The most important consideration to take into account for new diagnostic methods is that they should be as good as or better than the currently existing tools and, at the same time, be adequate for low-resource countries where the burden of TB is more important. For example NAA methods, especially the commercial kits that have the advantage of being well standardised and reproducible, have shown to be highly sensitive and specific in smear-positive samples; however, these values are much lower in smear-negative samples or in extrapulmonary specimens where the usefulness of these new 
tools would be much more desirable. The cost is another important consideration, since at the current prices these commercial kits are still out of the reach of most TB diagnostic laboratories in low-resource countries. Other molecular procedures even call for the use of sophisticated expensive equipment and highly-skilled personnel that are available only in developed countries or in central laboratory facilities in TB endemic countries. As long as these constraints are not properly addressed, expensive commercial kits making use of NAA techniques will remain restricted to developed countries or academic and research laboratories with the appropriate funding, but far away from of the TB control programmes.

Concerning serological approaches for the diagnosis of TB, none of the several tests proposed until now using a variety of mycobacterial antigens have shown to be predictive enough to warrant their routine use as a diagnostic test for TB. Apparently, tests using a cocktail of antigens, rather than a single more specific antigen, have given better results. The new ELISA-based tests like the QuantiFERON-TB test and the T SPOT-TB assay, which measure the production of IFN- $\gamma$ by activated $\mathrm{T}$ cells, are promising; however, more studies are needed in different settings to assess their usefulness as a diagnostic tool in certain populations, such as those subjects immunosuppresed by HIV infection or other diseases, and in children. The cost of these tests, since they are also available as a commercial kit, will have an impact on the feasibility for their implementation on a routine basis in the future.

The phage-based tests have also been evaluated in different settings either as a commercial kit or as the in-house method. The low sensitivity obtained in some of these studies could have been due to low infectivity of the phages, which also can be affected by the age and condition of the samples [35]. In contrast, in the studies where the phage-based methods have shown an increased sensitivity as compared with direct microscopy and culture, the volume of sample used was up to five-times higher than that used for culture. It seems that, in their current format, the phage-based assays are not ready as a tool to improve diagnosis of TB. However, they seem to be appropriate for rapid rifampicin resistance detection [73].

Many studies, in the search for newer and rapid TB diagnostic methods, are done with the hope of finding the "magic bullet", allowing diagnosis of TB in a matter of hours or on the spot. Maybe this should not be the way, and researchers should not rule out simple and pragmatic approaches that are closer to what is feasible to be implemented in TB diagnostic laboratories in high-endemic countries. For example, a simple technique as described by MEJIA et al. [114] and based on the rapid detection of micro-colonies of $M$. tuberculosis under a standard microscope, allowed detection of $>60 \%$ of the positive samples within the first 10 days, and after 2 weeks $>80 \%$ were positive with the micro-colony method compared to $10 \%$ on LJ medium. The same authors, in a report comprising $>1,800$ clinical samples, showed a sensitivity of $72 \%$ as compared with standard cultivation in LJ medium and conclude that the simultaneous use of both media increased sensitivity of detection [115]. Other recent approaches, like the one described by ANGEBY et al. [79], using the reduction of nitrate should be explored as a rapid diagnostic tool. Culture media incorporating potassium nitrate and rifampicin could be used directly on decontaminated sputum samples to detect not only $M$. tuberculosis, but also rifampicin-resistant bacilli at the same time. The same approach could be used with the recently described colorimetric methods, incorporating coloured indicators in the medium and inoculating directly with decontaminated sputum samples.

A final consideration is that any new method or approach, sophisticated or not, commercial or in-house, should be evaluated in well-designed and controlled clinical trials and tested in high-endemic, low-resource settings where the implementation and use of these methods are more needed to contribute to the improvement of tuberculosis control.

\section{REFERENCES}

1 World Health Organization. Global tuberculosis control: surveillance, planning, financing. WHO Report. Geneva, WHO/HTM/TB/2004.331, 2004.

2 Barnes PF, Lakey DL, Burman WJ. Tuberculosis in patients with HIV infection. Infect Dis Clin North Am 2002; 16: 107-126.

3 World Health Organization. Anti-tuberculosis drug resistance in the world. Report No. 2. Prevalence and trends. WHO/CDS/TB/2000.278, 2000.

4 Onyebujoh P, Rook GAW. Tuberculosis. Nat Rev Microbiol 2004; 2: 930-932.

5 Chan ED, Heifets L, Iseman MD. Immunologic diagnosis of tuberculosis: a review. Tuber Lung Dis 2000; 80: 131-140.

6 Takiff $\mathrm{H}$. The molecular mechanisms of drug resistance in Mycobacterium tuberculosis. In: Bastian I, Portaels F, eds. Multidrug-resistant tuberculosis. Dordrecht, The Netherlands, Kluwer Academic Publishers, 2000; pp. 77-114.

7 Foulds J, O'Brien R. New tools for the diagnosis of tuberculosis: the perspective of developing countries. Int J Tuberc Lung Dis 1998; 2: 778-783.

8 Andersen P, Munk ME, Pollock JM, Doherty TM. Specific immune-based diagnosis of tuberculosis. Lancet 2000; 356: 1099-1104.

9 Dockrell HM, Weir RE. Whole blood cytokine assays-a new generation of diagnostic tests for tuberculosis? Int $J$ Tuberc Lung Dis 1998; 6: 441-442.

10 Lalvani A. Spotting latent infection: the path to better tuberculosis control. Thorax 2003; 58: 916-918.

11 Pai M, Riley LW, Colford JM Jr. Interferon-gamma assays in the immunodiagnosis of tuberculosis: a systematic review. Lancet Infect Dis 2004; 4: 761-776.

12 Lein AD, Von Reyn CF. In vitro cellular and cytokine responses to mycobacterial antigens: application to diagnosis of tuberculosis infection and assessment of response to mycobacterial vaccines. Am J Med Sci 1997; 313: 364-371.

13 Brock I, Munk ME, Kok-Jensen A, Andersen P. Performance of whole blood IFN-gamma test for tuberculosis diagnosis based on PPD or the specific antigens ESAT-6 and CFP-10. Int J Tuberc Lung Dis 2001; 5: 462-467.

14 Angeby KA, Hoffner SE, Diwan VK. Should the 'bleach microscopy method' be recommended for improved case 
detection of tuberculosis? Literature review and key person analysis. Int J Tuberc Lung Dis 2004; 8: 806-815.

15 Ba F, Rieder HL. A comparison of fluorescence microscopy with the Ziehl-Neelsen technique in the examination of sputum for acid-fast bacilli. Int J Tuberc Lung Dis 1999; 3: 1101-1105.

16 Somoskövi Á, Hotaling JE, Fitzgerald M. Lessons from a proficiency testing event for acid-fast microscopy. Chest 2001; 120: 250-257.

17 Laszlo A. Tuberculosis: 7. Laboratory aspects of diagnosis. CMAJ 1999; 160: 1725-1729.

18 Tiruviluamala P, Reichman LB. Tuberculosis. Annu Rev Public Health 2002; 23: 403-426.

19 Kent TK, Kubica GP. Public Health mycobacteriology. A guide for level III laboratory. Atlanta, Centre for Disease Control, 1985.

20 Heifets L. Mycobacteriology laboratory. Clin Chest Med 1997; 18: 35-53.

21 Metchock BG, Nolte FS, Wallace RJ Jr. Mycobacterium. In: Murray PR, ed. Manual of Clinical Microbiology, 7th Edn. Washington DC, American Society for Microbiology, 1999; pp. 399-437.

22 Roberts GD, Goodman NL, Heifets L, et al. Evaluation of the BACTEC radiometric method for recovery of mycobacteria and drugsusceptibility testing of Mycobacterium tuberculosis from acid-fast smear-positive specimens. J Clin Microbiol 1983; 18: 689-696.

23 Hanna BA, Walters SB, Bonk SJ, Tick LJ. Recovery of mycobacteria from blood in mycobacteria growth indicator tube and Löwenstein-Jensen slant after lysiscentrifugation. J Clin Microbiol 1995; 33: 3315-3316.

24 Badak FZ, Kiska DL, Setterquist S, Hartley C, O'Connell MA, Hopfer RL. Comparison of mycobacteria growth indicator tube with BACTEC 460 for detection and recovery of mycobacteria from clinical specimens. $J$ Clin Microbiol 1996; 34: 2236-2239.

25 Pfyffer GE, Welscher HM, Kissling P, et al. Comparison of the Mycobacteria Growth Indicator Tube (MGIT) with radiometric and solid culture for recovery of acid-fast bacilli. J Clin Microbiol 1997; 35: 364-368.

26 Tortoli E, Cichero P, Piersimoni C, Simonetti MT, Gesu G, Nista D. Use of BACTEC MGIT 960 for recovery of mycobacteria from clinical specimens: multicentre study. J Clin Microbiol 1999; 37: 3578-3582.

27 Idigoras P, Beristain X, Iturzaeta A, Vicente D, PerezTrallero E. Comparison of the automated nonradiometric Bactec MGIT 960 system and Löwenstein-Jensen, Coletsos, and Middlebrook 7H11 solid media for recovery of mycobacteria. Eur J Clin Microb Infect Dis 2000; 19: 350-354.

28 Cambau E, Wichlacz C, Truffot-Pernot C, Jarlier V. Evaluation of the new MB redox system for detection of growth of mycobacteria. I Clin Microbiol 1999; 37: 2013-2015.

29 Rohner P, Ninet B, Metral C, Emler S, Auckenthaler R. Evaluation of the $\mathrm{MB} / \mathrm{BacT}$ system and comparison to the BACTEC 460 system and solid media for isolation of mycobacteria from clinical specimens. J Clin Microbiol 1997; 35: 3127-3131.

30 Woods GL, Fish G, Plaunt M, Murphy T. Clinical evaluation of difco ESP culture system II for growth and detection of mycobacteria. J Clin Microbiol 1997; 35: 121-124.

31 McNerney R. Phage replication technology for diagnosis and susceptibility testing. In: Parish T, Stocker NG, eds. Mycobacterium tuberculosis protocols. Methods in Molecular Medicine., Totowa, NY, Humana Press, 2001; pp. 145-154.

32 Albert H, Heydenrych A, Brookes R, et al. Performance of a rapid phage-based test, FASTPlaqueTB, to diagnose pulmonary tuberculosis from sputum specimens in South Africa. Int J Tuberc Lung Dis 2002; 6: 529-537.

33 Muzaffar R, Batool S, Aziz F, Naqvi A, Rizvi A. Evaluation of the FASTPlaqueTB assay for direct detection of Mycobacterium tuberculosis in sputum specimens. Int J Tuberc Lung Dis 2002; 6: 635-640.

34 Takiff $\mathrm{H}$, Heifets $\mathrm{L}$. In search of rapid diagnosis and drug-resistance detection tools: is the FASTPlaqueTB test the answer? Int J Tuberc Lung Dis 2002; 6: 560-561.

35 Mbulo GM, Kambashi BS, Kinkese J, et al. Comparison of two bacteriophage tests and nucleic acid amplification for the diagnosis of pulmonary tuberculosis in sub-Saharan Africa. Int J Tuberc Lung Dis 2004; 8: 1342-1347.

36 Carriere C, Riska PF, Zimhony O, et al. Conditionally replicating luciferase reporter phages: improved sensitivity for rapid detection and assessment of drug susceptibility of Mycobacterium tuberculosis. I Clin Microbiol 1997; 35: 3232-3239.

37 Banaiee N, Bobadilla-Del-Valle M, Bardarov S Jr, et al. Luciferase reporter mycobacteriophages for detection, identification, and antibiotic susceptibility testing of Mycobacterium tuberculosis in Mexico. J Clin Microbiol 2001; 39: 3883-3888.

38 Butler WR, Guthertz LS. Mycolic acid analysis by highperformance liquid chromatography for identification of Mycobacterium species. Clin Microbiol Rev 2001; 14: 704-726.

39 Evans KD, Nakasone AS, Sutherland PA, de la Maza LM, Peterson EM. Identification of Mycobacterium tuberculosis and Mycobacterium avium-M. intracellulare directly from primary BACTEC cultures by using acridinium-ester labelled DNA probes. J Clin Microbiol 1992; 30: 2427-2431.

40 Miller N, Infante S, Cleary T. Evaluation of the LiPA MYCOBACTERIA assay for identification of mycobacterial species from BACTEC 12B bottles. J Clin Microbiol 2000; 38: 1915-1919.

41 Suffys PN, da Silva Rocha A, de Oliveira M, et al. Rapid identification of Mycobacteria to the species level using INNO-LiPA Mycobacteria, a reverse hybridization assay. J Clin. Microbiol 2001; 39: 4477-4482.

42 Ruiz P, Gutierrez J, Zerolo FJ, Casal M. GenoType mycobacterium assay for identification of mycobacterial species isolated from human clinical samples by using liquid medium. J Clin Microbiol 2002; 40: 3076-3078.

43 Stender $\mathrm{H}$, Lund $\mathrm{K}$, Petersen $\mathrm{KH}$, et al. Fluorescence in situ hybridization assay using peptide nucleic acid probes for differentiation between tuberculous and nontuberculous mycobacterium species in smears of mycobacterium cultures. I Clin Microbiol 1999; 37 2760-2765.

44 Zerbi P, Schonau A, Bonetto S, et al. Amplified in situ hybridization with peptide nucleic acid probes for 
differentiation of Mycobacterium tuberculosis complex and nontuberculous Mycobacterium species on formalin-fixed, paraffin-embedded archival biopsy and autopsy samples. Am J Clin Pathol 2001; 116: 770-775.

45 Rogall T, Flohr T, Bottger EC. Differentiation of Mycobacterium species by direct sequencing of amplified DNA. J Gen Microbiol 1990; 136: 1915-1920.

46 Kirschner P, Springer B, Vogel U, et al. Genotypic identification of mycobacteria by nucleic acid sequence determination: report of a 2-year experience in a clinical laboratory. J Clin Microbiol 1993; 31: 2882-2889.

47 Soini $\mathrm{H}$, Bottger EC, Viljanen MK. Identification of mycobacteria by PCR-based sequence determination of the 32-kilodalton protein gene. J Clin Microbiol 1994; 32: 2944-2947.

48 Roth A, Fischer M, Hamid ME, Michalke S, Ludwig W, Mauch H. Differentiation of phylogenetically related slowly growing mycobacteria based on 16S-23S rRNA gene internal transcribed spacer sequences. I Clin Microbiol 1998; 36: 139-147.

49 Telenti A, Marchesi F, Balz M, Bally F, Bottger EC, Bodmer T. Rapid identification of mycobacteria to the species level by polymerase chain reaction and restriction enzyme analysis. J Clin Microbiol 1993; 31: 175-178.

50 da Silva Rocha A, da Costa Leite C, Torres HM, et al. Use of PCR-restriction fragment length polymorphism analysis of the hsp65 gene for rapid identification of mycobacteria in Brazil. J Microbiol Methods 1999; 37: 223-229.

51 Ergin A, Kocagoz T, Us D. Evaluation of 120 mycobacterial strains isolated from clinical specimens to the species level by polymerase chain reaction-restriction enzyme analysis. Scand J Infect Dis 2000; 32: 657-662.

52 Gingeras TR, Ghandour G, Wang E, et al. Simultaneous genotyping and species identification using hybridization pattern recognition analysis of generic Mycobacterium DNA arrays. Genome Res 1998; 8: 435-448.

53 Troesch A, Nguyen H, Miyada CG, et al. Mycobacterium species identification and rifampin resistance testing with high-density DNA probe arrays. J Clin Microbiol 1999; 37: 49-55.

54 Canetti G, Fox W, Khomenko A, et al. Advances in techniques of testing mycobacterial drug sensitivity, and the use of sensitivity tests in tuberculosis control programmes. Bull World Health Organ 1969; 41: 21-43.

55 Siddiqi SH, Libonati JP, Middlebrook G. Evaluation of rapid radiometric method for drug susceptibility testing of Mycobacterium tuberculosis. J Clin Microbiol 1981; 13: 908-912.

56 Heifets LB, Cangelosi GA. Drug susceptibility testing of Mycobacterium tuberculosis: a neglected problem at the turn of the century. Int J Tuberc Lung Dis 1999; 3: 564-581.

57 Cooksey RC, Morlock GP, McQueen A, Glickman SE, Crawford JT. Characterisation of streptomycin resistance mechanisms among Mycobacterium tuberculosis isolates from patients in New York City. Antimicrob Agents Chemother 1996; 40: 1186-1188.

58 Delgado MB, Telenti A. Detection of mutations associated with quinolone resistance in Mycobacterium tuberculosis. In: Persing DH, ed. Selected PCR protocols for emerging infectious diseases. Washington DC, American Society for Microbiology, 1996; pp. 138-143.

59 Telenti A, Imboden P, Marchesi F, et al. Detection of rifampicin-resistance mutations in Mycobacterium tuberculosis. Lancet 1993; 341: 647-650.

60 Takiff HE, Salazar L, Guerrero C, et al. Cloning and nucleotide sequence of Mycobacterium tuberculosis gyrA and gyrB genes and detection of quinolone resistance mutations. Antimicrob Agents Chemother 1994; 38: 773-780.

61 Mestdagh M, Fonteyne PA, Realini L, et al. Relationship between pyrazinamide resistance, loss of pyrazinamidase activity, and mutations in the pncA locus in multidrugresistant clinical isolates of Mycobacterium tuberculosis. Antimicrob Agents Chemother 1999; 43: 2317-2319.

62 Williams DL, Waguespack C, Eisenach K, et al. Characterization of rifampin-resistance in pathogenic mycobacteria. Antimicrob Agents Chemother 1994; 38: 2380-2386.

63 De Beenhouwer H, Lhiang Z, Jannes G, et al. Rapid detection of rifampicin resistance in sputum and biopsy specimens from tuberculosis patients by PCR and line probe assay. Tuberc Lung Dis 1995; 76: 425-430.

64 Rossau R, Traore H, De Beenhouwer H, et al. Evaluation of the INNO-LiPA Rif. TB assay, a reverse hybridization assay for the simultaneous detection of Mycobacterium tuberculosis complex and its resistance to rifampin. Antimicrob Agents Chemother 1997; 41: 2093-2098.

65 Sougakoff $\mathrm{W}$, Rodriguez M, Truffot-Pernot C, et al. Use of a high-density DNA probe array for detecting mutations involved in rifampicin resistance in Mycobacterium tuberculosis. Clin Microbiol Infect 2004; 10: 289-294.

66 Ruiz M, Torres MJ, Llanos AC, Arroyo A, Palomares JC, Aznar J. Direct detection of rifampin- and isoniazidresistant Mycobacterium tuberculosis in auraminerhodamine-positive sputum specimens by real-time PCR. J Clin Microbiol 2004; 42: 1585-1589.

67 Palaci M, Ueki SY, Sato DN, et al. Evaluation of mycobacteria growth indicator tube for recovery and drug susceptibility testing of Mycobacterium tuberculosis isolates from respiratory specimens. J Clin Microbiol 1996; 34: 762-764.

68 Palomino JC, Traore H, Fissette K, Portaels F. Evaluation of Mycobacteria Growth Indicator Tube (MGIT) for drug susceptibility testing of Mycobacterium tuberculosis. Int J Tuberc Lung Dis 1999; 3: 344-348.

69 Sanders CA, Nieda RR, Desmond EP. Validation of the use of Middlebrook 7H10 agar, BACTEC MGIT 960, and BACTEC 460 12B media for testing the susceptibility of Mycobacterium tuberculosis to levofloxacin. J Clin Microbiol 2004; 42: 5225-5228.

70 Hausdorfer J, Sompek E, Allerberger F, Dierich MP, Rusch-Gerdes S. E-test for susceptibility testing of Mycobacterium tuberculosis. Int J Tuberc Lung Dis 1998; 2: 751-755.

71 Tortoli E, Mattei R, Savarino A, Bartolini L, Beer J. Comparison of Mycobacterium tuberculosis susceptibility testing performed with BACTEC 460TB (Becton Dickinson) and MB/BacT (Organon Teknika) systems. Diagn Microbiol Infect Dis 2000; 38: 83-86.

72 Ruiz P, Zerolo FJ, Casal MJ. Comparison of susceptibility testing of Mycobacterium tuberculosis using the ESP culture 
system II with that using the BACTEC method. J Clin Microbiol 2000; 38: 4663-4664.

73 Simboli N, Takiff H, McNerney R, et al. In-house phage amplification assay is a sound alternative for detecting rifampin-resistant Mycobacterium tuberculosis in lowresource settings. Antimicrob Agents Chemother 2005; 49: 425-427.

74 Albert H, Trollip A, Seaman T, Mole RJ. Simple, phagebased (FASTPplaque) technology to determine rifampicin resistance of Mycobacterium tuberculosis directly from sputum. Int J Tuberc Lung Dis 2004; 8: 1114-1119.

75 Palomino JC, Martin A, Camacho M, Guerra H, Swings J, Portaels F. Resazurin microtiter assay plate: simple and inexpensive method for detection of drug resistance in Mycobacterium tuberculosis. Antimicrob Agents Chemother 2002; 46: 2720-2722.

76 Yajko DM, Madej JJ, Lancaster MV, et al. Colorimetric method for determining MICs of antimicrobial agents for Mycobacterium tuberculosis. J Clin Microbiol 1995; 33: 2324-2327.

77 Abate G, Mshana RN, Miorner H. Evaluation of a colorimetric assay based on 3-(4,5-dimethylthiazol-2-yl)2,5-diphenyl tetrazolium bromide (MTT) for rapid detection of rifampicin resistance in Mycobacterium tuberculosis. Int J Tuberc Lung Dis 1998; 2: 1011-1016.

78 Martin A, Camacho M, Portaels F, Palomino JC. Resazurin microtiter assay plate testing of Mycobacterium tuberculosis susceptibilities to second-line drugs: rapid, simple, and inexpensive method. Antimicrob Agents Chemother 2003; 47: 3616-3619.

79 Angeby KA, Klintz L, Hoffner SE. Rapid and inexpensive drug susceptibility testing of Mycobacterium tuberculosis with a nitrate reductase assay. J Clin Microbiol 2002; 40: 553-555.

80 Montoro E, Lemus D, Echemendia M, Martin A, Portaels F, Palomino JC. Comparative evaluation of the nitrate reduction assay, the MTT test, and the resazurin microtitre assay for drug susceptibility testing of clinical isolates of Mycobacterium tuberculosis. I Antimicrob Chemother 2005; 55: 500-505.

81 Martin A, Palomino JC, Portaels F. Rapid detection of ofloxacin resistance in Mycobacterium tuberculosis by two low-cost colorimetric methods: resazurin and nitrate reductase assays. J Clin Microbiol 2005; 43: 1612-1616.

82 Sandin RL. Polymerase chain reaction and other amplification techniques in mycobacteriology. Clin Lab Med 1996; 16: 617-633.

83 Kearns AM, Freeman R, Steward M, Magee JG. A rapid polymerase chain reaction technique for detecting $M$. tuberculosis in a variety of clinical specimens. J Clin Pathol 1998; 51: 922-924.

84 Noordhoek GT, van Embden JD, Kolk, AH. Reliability of nucleic acid amplification for detection of Mycobacterium tuberculosis: an international collaborative quality control study among 30 laboratories. J Clin Microbiol 1996; 34: 2522-2525.

85 Suffys P, Palomino JC, Cardoso Leão S, et al. Evaluation of the polymerase chain reaction for the detection of Mycobacterium tuberculosis. Int J Tuberc Lung Dis 2000; 4: 179-183.
86 Kwoh DY, Davis GR, Whitfield KM, Chappelle HL, DiMichele LJ, Gingeras TR. Transcription-based amplification system and detection of amplified human immunodeficiency virus type 1 with a bead-based sandwich hybridization format. Proc Natl Acad Sci USA 1989; 86: 1173-1177.

87 Abe C, Hirano K, Wada M, et al. Detection of Mycobacterium tuberculosis in clinical specimens by polymerase chain reaction and Gen-Probe amplified mycobacterium tuberculosis direct test. J Clin Microbiol 1993; 31: 3270-3274.

88 Dalovisio JR, Montenegro-James S, Kemmerly SA. Comparison of the Amplified Mycobacterium tuberculosis (MTB) direct test, Amplicor MTB PCR, and IS6110PCR for detection of MTB in respiratory specimens. Clin Infect Dis 1996; 23: 1099-1106.

89 CDC. Nucleic acid amplification tests for tuberculosis. MMWR 2000; 49: 593-594.

90 Rajalahti I, Vuorinen P, Liippo K, Nieminen MM, Miettinen A. Evaluation of commercial DNA and rRNA amplification assays for assessment of treatment outcome in pulmonary tuberculosis patients. Eur J Clin Microbiol Infect Dis 2001; 20: 746-750.

91 Pfyffer GE. Laboratory diagnosis of tuberculosis. In: Kaufmann SHE, Hahn H, eds. Mycobacteria and TB. Basel, Karger, Switzerland, 2003; pp. 67-83.

92 Shamputa IC, Rigouts L, Portaels F. Molecular genetic methods for diagnosis and antibiotic resistance detection of mycobacteria from clinical specimens. APMIS 2004; 112: 728-752.

93 Broccolo F, Scarpellini P, Locatelli G, et al. Rapid diagnosis of mycobacterial infections and quantitation of Mycobacterium tuberculosis load by two real-time calibrated PCR assays. J Clin Microbiol 2003; 41: 4565-4572.

94 Bergmann JS, Woods GL. Clinical evaluation of the BDProbeTec strand displacement amplification assay for rapid diagnosis of tuberculosis. J Clin Microbiol 1998; 36: 2766-2768.

95 Pfyffer GE, Funke-Kissling P, Rundler E, Weber R. Performance characteristics of the BDProbeTec system for direct detection of Mycobacterium tuberculosis complex in respiratory specimens. J Clin Microbiol 1999; 37: 137-140.

96 Bergmann JS, Keating WE, Woods GL. Clinical evaluation of the BDProbeTec ET system for rapid detection of Mycobacterium tuberculosis. J Clin Microbiol 2000; 38: 863-865.

97 van Embden JD, Cave MD, Crawford JT, et al. Strain identification of Mycobacterium tuberculosis by DNA fingerprinting: recommendations for a standardized methodology. J Clin Microbiol 1993; 31: 406-409.

98 Kamerbeek J, Schouls L, Kolk A, et al. Simultaneous detection and strain differentiation of Mycobacterium tuberculosis for diagnosis and epidemiology. J Clin Microbiol 1997; 35: 907-914.

99 van Soolingen D, de Haas PE, Hermans PW, Groenen PM, van Embden JD. Comparison of various repetitive DNA elements as genetic markers for strain differentiation and epidemiology of Mycobacterium tuberculosis. J Clin Microbiol 1993; 31: 1987-1995. 
100 Goguet de la Salmoniere YO, Kim CC, Tsolaki AG, Pym AS, Siegrist MS, Small PM. High-throughput method for detecting genomic-deletion polymorphisms. J Clin Microbiol 2004; 42: 2913-2918.

101 Supply P, Lesjean S, Savine E, Kremer K, van Soolingen D, Locht C. Automated high-throughput genotyping for study of global epidemiology of Mycobacterium tuberculosis based on mycobacterial interspersed repetitive units. J Clin Microbiol 2001; 39: 3563-3571.

102 Cowan LS, Mosher L, Diem L, Massey JP, Crawford JT. Variable-number tandem repeat typing of Mycobacterium tuberculosis isolates with low copy numbers of IS6110 by using mycobacterial interspersed repetitive units. J Clin Microbiol 2002; 40: 1592-1602.

103 Kanduma E, McHugh TD, Gillespie SH. Molecular methods for Mycobacterium tuberculosis strain typing: a user's guide. J Appl Microbiol 2003; 94: 781-791.

104 Maekura R, Okuda Y, Nakagawa M, et al. Clinical evaluation of anti-tuberculous glycolipid immunoglobulin $\mathrm{G}$ antibody assay for rapid serodiagnosis of pulmonary tuberculosis. J Clin Microbiol 2001; 39: 3603-3608.

105 Gounder C, De Queiroz Mello FC, Conde MB, et al. Field evaluation of a rapid immunochromatographic test for tuberculosis. J Clin Microbiol 2002; 40: 1989-1993.

106 Grange JM, Laszlo A. Serodiagnostic tests for tuberculosis: a need for assessment of their operational predictive accuracy and acceptability. Bull World Health Organ 1990; 68: 571-576.

107 Lyashchenko KP, Singh M, Colangeli R, Gennaro ML. A multi-antigen print immunoassay for the development of serological diagnosis of infectious diseases. I Immunol Methods 2000; 28: 91-100.

108 Gennaro ML. Immunologic diagnosis of tuberculosis. Clin Infect Dis 2000; 30: Suppl. 3, S243-S246.

109 Kataria YP, Khurshid I. Adenosine deaminase in the diagnosis of tuberculous pleural effusion. Chest 2001; 120: 334-336.

110 Lee YC, Rogers JT, Rodriguez RM, Miller KD, Light RW. Adenosine deaminase levels in nontuberculous lymphocytic pleural effusions. Chest 2001; 120: 356-361.

111 Corral I, Quereda C, Navas E, et al. Adenosine deaminase activity in cerebrospinal fluid of HIV-infected patients: limited value for diagnosis of tuberculous meningitis. Eur J Clin Microbiol Infect Dis 2004; 23: 471-476.

112 Conde MB, Marinho SR, Pereira Mde F, et al. The usefulness of serum adenosine deaminase 2 (ADA2) activity in adults for the diagnosis of pulmonary tuberculosis. Respir Med 2002; 96: 607-610.

113 Lima DM, Colares JK, da Fonseca BA. Combined use of the polymerase chain reaction and detection of adenosine deaminase activity on pleural fluid improves the rate of diagnosis of pleural tuberculosis. Chest 2003; 124: 909-914.

114 Mejia GI, Castrillon L, Trujillo H, Robledo JA. Microcolony detection in 7H11 thin layer culture is an alternative for rapid diagnosis of Mycobacterium tuberculosis infection. Int J Tuberc Lung Dis 1999; 3: 138-142.

115 Mejia GI, Guzman A, Agudelo CA, Trujillo H, Robledo J. Five year experience with thin layer agar medium for rapid diagnosis of tuberculosis. Biomedica 2004; 24: Suppl. $1,52-59$. 